\title{
Different types of repeat expansion in the TATA-binding protein gene are associated with a new form of inherited ataxia
}

\author{
Christine Zühlke ${ }^{*}, 1$, Yorck Hellenbroich ${ }^{1,4}$, Andreas Dalski ${ }^{1,4}$, Nina Kononowa ${ }^{1}$, \\ Johann Hagenah ${ }^{2}$, Peter Vieregge ${ }^{2}$, Olaf Riess ${ }^{3}$, Christine Klein ${ }^{2}$ and Eberhard Schwinger ${ }^{1}$ \\ ${ }^{1}$ Institut für Humangenetik, Universität Lübeck, 23538 Lübeck, Germany; ${ }^{2}$ Klinik für Neurologie, Universität \\ Lübeck, 23538 Lübeck, Germany; ${ }^{3}$ Universität Rostock, Medizinische Genetik, 18055 Rostock, Germany
}

\begin{abstract}
A novel neurological syndrome has recently been described to be associated with an expanded polyglutamine domain. The expansion results from partial duplication within the TATA-binding protein (TBP). By investigation of 604 sporadic and familial cases with various forms of neurological syndromes and 157 unaffected individuals, we found repeat expansions in the TBP in four patients of two families with autosomal dominant inheritance of ataxia, dystonia, and intellectual decline. Two different genotypes for the repetitive sequence could be demonstrated which led to elongated polyglutamine stretches between 50 and 55 residues, whereas normal alleles with $\mathbf{2 7}$ to a maximum of $\mathbf{4 4}$ glutamine residues were found in this study. The expansion to $\mathbf{5 0}$ or more glutamine residues results in a pathological phenotype and confirms the report of a new polyglutamine disease. European Journal of Human Genetics (2001) 9, 160-164.
\end{abstract}

Keywords: TATA-binding protein; CAG repeat expansion; polyglutamine disease; hereditary ataxia; dystonia

\section{Introduction}

Unstable expansions of trinucleotide repeats have been shown to cause at least 14 neurological diseases (for review: $\left.{ }^{1}\right)$. The growing number of inherited disorders associated with this kind of mutation has led researchers to study polymorphic repeats in known genes. Gostout and coworkers ${ }^{2}$ analysed a highly polymorphic ${ }^{3}$ imperfect repeat in the gene for the TATA-binding protein (TBP), a general transcription initiation factor that regulates the expression of most eukaryotic genes transcribed by RNA polymerase II. ${ }^{4-6}$ They found 20 different alleles with 29 to 40 glutamine residues in the amino-terminal region of the protein. Furthermore, they characterised the allelic

\footnotetext{
*Correspondence: C Zühlke, Institut für Humangenetik, Med. Universität Lubeck, Ratzeburger Allee 160, 23538 Lübeck, Germany

Tel: +49 451500 2621; Fax: +49 451500 4187;

E-mail: zuehlke@medinf.mu-luebeck.de

${ }^{4}$ These authors contributed equally to this work.

Received 24 October 2000; revised 24 November 2000; accepted
} 25 November 2000 distribution among various human populations and studied the repeat sequences in primates, mouse, and drosophila. The most common human alleles encode 32 to 39 glutamines and the longest allele harbours 42 repeats. ${ }^{7}$ The polyglutamine-encoding DNA sequence can be divided into five regions including two polymorphic $(\mathrm{CAG})_{\mathrm{n}}$ stretches: $^{2}(\mathrm{CAG})_{3}(\mathrm{CAA})_{3} \cdot(\mathrm{CAG})_{\mathrm{n}}$. CAA CAG CAA. $(\mathrm{CAG})_{\mathrm{n}} \cdot \mathrm{CAA}$ CAG.

Assuming a further association between repeat expansions and neurological diseases, the TBP gene has been investigated intensively as a candidate locus. Among 118 patients with various forms of neurological diseases studied, one individual showed a de novo expansion of the repeat in the TBP gene due to a partial duplication, ${ }^{8}$ resulting in an expanded polyglutamine domain. Disorders caused by polyglutamine repeats have similar features and possibly share common mechanisms of pathogenesis. All known disorders in this group are characterised by progressive neurological dysfunction that typically begins in early adulthood and results in neurodegeneration, genetic anticipation, and translation of the repetitive sequence into a polyglutamine stretch in the 
encoded protein. The autosomal dominantly inherited diseases display characteristic, but partially overlapping, clinical and pathological findings. The phenotype and severity of these disorders can vary greatly, primarily depending on the repeat length.

To date, only one case with an expanded polyglutamine stretch has been described for the TBP. ${ }^{8}$ No data concerning repeat instability or mode of inheritance are available as yet. In the present study we report the analysis of 604 patients with neurological symptoms in whom expansions of previously identified genes had been excluded, and 157 healthy control individuals of German origin. We investigated the allelic distribution and allele frequency and found repeat expansion in the TBP gene in two of our families with autosomal dominant inheritance.

\section{Patients and methods Subjects}

We studied 604 patients with ataxia and gait disturbances in whom repeat expansions of previously identified genes had been excluded and 157 unaffected individuals of German origin. In the patient group we screened 135 unrelated individuals with dominantly inherited neurologic disorders (symptoms of ataxia and gait disturbances) and 469 with idiopathic sporadic ataxia. After having obtained informed consent for genetical analyses, total genomic DNA was extracted from peripheral blood leukocytes by standard protocols.

\section{PCR analysis of the CAG repeats in the TBP gene}

For analysis of repeat lengths, PCR was performed in a volume of $25 \mu \mathrm{l}$ containing $50 \mathrm{ng}$ genomic DNA, $10 \mathrm{pmol}$ of each primer, 5 pmol dNTP and 1 unit Taq polymerase (QBiogene) with an annealing temperature of $62^{\circ} \mathrm{C}$ using primers described by Koide et al. ${ }^{8}$ Products were separated on $2.0 \%$ agarose gels and visualised by ethidium bromide staining followed by separation on $6 \%$ denaturing polyacrylamide gels and silver staining. Allele sizes were estimated by comparison to molecular weight markers and M13 sequencing ladders.

\section{Sequence analysis}

To determine the nucleotide sequence and size of normal and expanded TBP alleles, PCR products were cloned into the vector pCR 2.1-TOPO (Invitrogen) and sequenced using the dideoxy chain termination method on double-stranded DNA templates in the presence of IRD800-labelled primers on a Licor 4200 automated sequencer. For each template, up to 10 single colonies have been analysed to detect the most representative repeat length. Due to somatic mosaicism or instability of repetitive sequences in bacteria, the repeat length may vary by \pm 1 unit between the subclones. Variability is frequent in expanded alleles but is the exception within the normal range.

\section{Case reports}

Family I

Case $I_{1}$ A 47-year old former house wife presented with a 13-year history of slowly progressive ataxia, dystonic posturing of the feet, mood and personality changes, and mild deterioration of intellectual function. Over the past year she also developed marked spasticity of all four extremities, an akinetic-rigid parkinsonian syndrome following haloperidol therapy, urinary incontinence, and, most recently, complete cessation of speech production. The patient was the product of a normal pregnancy and delivery; early milestones and general health were completely normal up to the age of 34 years. The patient is married and has three daughters aged 16, 21, and 23 years. The eldest daughter was also diagnosed with a neurological syndrome (Case $\mathrm{I}_{2}$ ). According to her father, the second daughter was noted to develop torticollis aged 20 years, while the youngest daughter appeared unaffected (both unavailable for neurologic examination). The index patient's mother was affected by an unexplained gait disorder (by history), and her sister $\left(\mathrm{II}_{3}\right)$ is also affected by cerebellar ataxia. On her most recent neurologic examination, the patient was awake but mute, showed saccadic gaze, marked cerebellar ataxia, and spasticity of all four extremities which were held in a flexed posture with beginning contractures. Reflexes were brisk bilaterally but Babinski's sign was negative. The patient was bedridden and could not sit, stand or walk. The sensory system appeared normal. Serum analyses of copper, ceruloplasmin, thyroxine, and lactate levels were normal, as were the results of blood counts and smears. Molecular genetic testing for known CAG repeat expansions was negative. A brain CT scan showed generalised cerebral atrophy, most pronounced in the cerebellar region.

Case $I_{2}$ This 23-year old woman, the daughter of patient $\mathrm{I}_{1}$, was also the product of a normal pregnancy and delivery. She reported a 3-year history of writing problems due to involuntary flexion of the fingers of her right hand. On neurological examination she did not have any obvious impairment of intellectual function but appeared somewhat slow and clumsy. Cranial nerve examination was unremarkable except for scandic speech, mild head ataxia, and torticollis. Tone was increased in all four extremities, reflexes were normal and pyramidal tract signs absent. Coordination and fine motor function were impaired bilaterally, and she showed dysmetria and severe dysdiadochokinesia, more pronounced on the right hand side. On writing, the patient displayed dystonia of the right hand (writer's cramp) with coinnervation of the left hand. Laboratory results were unrevealing, as was molecular analysis for SCA1, 2, 3, 6, 7, 8 , and DRPLA. An MRI scan of the head was normal.

Case $\boldsymbol{I}_{3}$ This 49-year old woman (sister of Case $\mathrm{I}_{1}$ ) had an ataxic syndrome according to medical records but was not personally seen by us. A DNA sample was available. 


\section{Family II}

Case II This 21-year-old man had an 8-year history of deteriorating intellectual function, followed by gait and speech problems, general clumsiness of movements, and recent urinary incontinence. By history, his mother, maternal grandmother, her grandmother's paternal uncle and niece were also affected by a similar neurological syndrome, again indicating autosomal dominant inheritance. On examination, the patient had dysarthria and decreased speech production. He showed pathological saccades, horizontal gaze-evoked nystagmus, postural and intention tremor of both arms, increased tone of all four extremities, and bradydysdiadochokinesia of the right and adiadochokinesia of the left hand. Reflexes were brisk bilaterally, he had an extensor plantar response on the right and an ataxic gait. The sensory system appeared normal. Laboratory results were unremarkable; an MRI scan of the head showed marked cerebellar and mild brain stem atrophy.

Case III Positive control of a patient with ataxia (O Riess, unpublished data) and repeat expansion in the TBP gene.

\section{Results and Discussion}

\section{Expanded alleles in TBP}

We analysed 469 patients with sporadic ataxia and 135 unrelated cases with inherited ataxias for repeat sizes in the TBP gene. Among the 135 inherited cases with ataxia, we found two families with repeat expansions. Both families (I (three patients available) and II (one patient available)) were of northern German extraction and displayed autosomal dominant inheritance of the disorder (frequency 2: 135 families with non-SCA 1-12). Results, including a positive control, are shown in Figure 1A. The expanded alleles ranged between 50 and 55 glutamine codons as determined by direct sequencing and are associated with the following haplotypes:

- $\mathrm{I}_{1}(\mathrm{CAG})_{3}(\mathrm{CAA})_{3}(\mathrm{CAG})_{45} \mathrm{CAA} \mathrm{CAG}$;

- $\mathrm{I}_{2}(\mathrm{CAG})_{3}(\mathrm{CAA})_{3}(\mathrm{CAG})_{47} \mathrm{CAA} \mathrm{CAG}$;

- $\mathrm{I}_{3}(\mathrm{CAG})_{3}(\mathrm{CAA})_{3}(\mathrm{CAG})_{46} \mathrm{CAA} \mathrm{CAG}$;

- II $(\mathrm{CAG})_{3}(\mathrm{CAA})_{3}(\mathrm{CAG})_{9}$ CAA CAG CAA (CAG) $)_{31}$ CAA CAG;

- III (CAG) $)_{3}(\mathrm{CAA})_{3}(\mathrm{CAG})_{9}$ CAA CAG CAA (CAG) $)_{30}$ CAA CAG.

In Family I, the complex repeat structure is reduced as characterised by loss or mutation of interrupting CAA triplets. Consequently, an instability of the expanded allele could be observed. The affected sisters $\mathrm{I}_{1}$ and $\mathrm{I}_{3}$ differ by one trinucleotide repeat in the TBP gene. Upon transmission from mother $\left(\mathrm{I}_{1}\right)$ to daughter $\left(\mathrm{I}_{2}\right)$, the repeat was elongated by two units from 53 to 55 repeats. Possibly, this expansion may contribute to the earlier age of onset in the daughter (see below). In Cases II and III, the CAA CAG CAA element following the first polymorphic (CAG) repeat is still present but is followed by a CAG expansion in comparison to normal alleles with a maximum of 21 triplets at this site. ${ }^{7}$ Analysis of these expansions in relatives, who are currently unavailable, might give information concerning the meiotic stability of these genotypes.

In our patients the shortest allele correlating with a disease phenotype comprises 50 coding triplets, whereas the longest normal allele has 44 . Therefore, the gap between the normal and pathological repeat length is at a maximum of five triplets. No repeat expansion in the TBP gene was detected in any of the sporadic patients.

\section{Distribution of TBP normal alleles in unaffected} individuals and in sporadic and familial ataxia patients In order to define the range of the repeat length in normal TBP alleles, we investigated 157 control subjects (314 chromosomes) who did not present with any neurological or psychiatric symptoms at the time of blood sampling. Additionally, there was no known family history of any neurological disorder in the control group. PCR products were separated on PAA gels as shown in Figure 1B.

Fifteen different normal alleles have been identified, ranging from 27 to 44 glutamine codons (Table 1). The most frequent allele 5 (40\%) consists of 38 triplets, followed by allele $6(22 \%)$ with 37 repeats, and allele 7 (17\%) with 36 repeats. The repeat number and composition of eleven unrelated normal alleles was determined by sequence analysis revealing four different haplotypes. The size of the remaining alleles was calculated by comparison to a sequencing ladder.

The distribution and frequency of normal alleles differs between the German and Japanese ${ }^{8}$ samples, with slightly longer normal alleles in the German population. This may be due to different ancestors leading to differential allelic distribution among the various populations. ${ }^{2}$ It remains to be investigated whether this fact causes a higher frequency of the disease in Europe, especially in northern Germany.

By comparing the distribution of unexpanded alleles in affected individuals with that of the control sample, we found no significant differences (Table 1). Neither frequency nor ranges varied between the groups. Homozygosity for the most abundant alleles was found in approximately $23 \%$ of investigated subjects. The composition of interrupted normal alleles was $(\mathrm{CAG})_{3}(\mathrm{CAA})_{3}(\mathrm{CAG})_{\mathrm{nI}}$ CAA CAG CAA $(\mathrm{CAG})_{\mathrm{nI}}$ CAA CAG with $\mathrm{nI}=9$ in eight of 11 sequenced alleles and with nII being highly polymorphic.

In summary, we describe a novel neurological syndrome with predominant ataxia (Patients $\mathrm{I}_{1}, \mathrm{I}_{2}, \mathrm{II}$ ) but also involvement of the pyramidal (probably all three patients), extrapyramidal (Family I) and possibly autonomic system (urinary incontinence in Patient $\mathrm{I}_{1}$ and II). In addition, intellectual impairment and cessation of speech production point to a degenerative multisystem disorder. However, marked intra- and interfamilial phenotypic variability was observed. Our patients had adolescent to young adult onset 
a

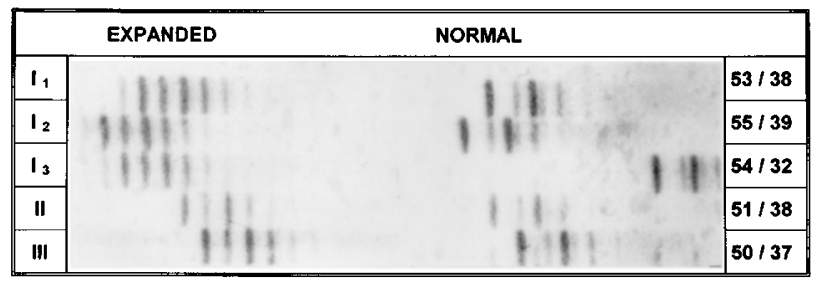

b

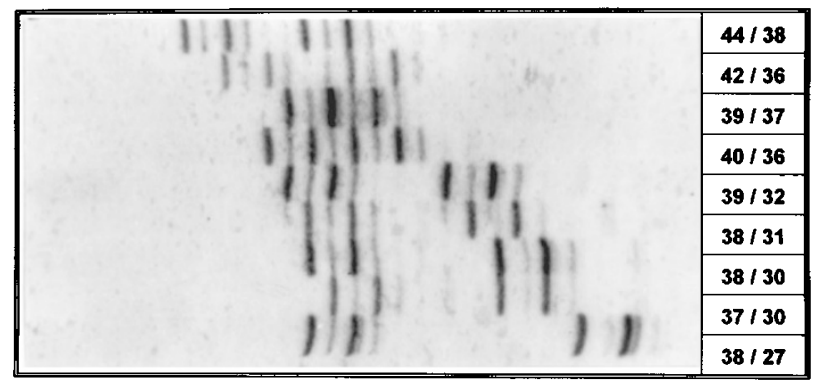

Figure 1 PCR amplification of DNA from control individuals and affected individuals. Products were resolved on denaturing polyacrylamide gels and detected by silver staining. Numbers of glutamine triplets are given. (A) Results for five DNA samples with expanded alleles ranging from 50 to 55 repeats. DNA samples $\mathrm{I}_{1-3}$ and II are familial cases, sample III is a positive control. (B) Products of nine individuals with alleles in the normal range.

(12 to 34 years) in contrast to the childhood onset in the Japanese patient which may be due to the higher number of 62 glutamine coding triplets in the TBP gene. Furthermore, our patients had no muscle weakness, epilepsy or short stature. Similar to the Japanese patient, our patients displayed a cerebellar syndrome, spasticity (Cases $\mathrm{I}_{1}$ and II), extensor plantar response (Case II), impaired intellectual function in Case II and to a much lesser extent in Family I, as well as cerebellar and milder cerebral atrophy. Our patients show some clinical overlap mainly with three other neurodegenerative disorders SCA2, SCA3 and DRPLA ${ }^{9-11}$ are visible. The identification of additional cases should help to better define the phenotypic spectrum and genetic correlations of this novel entity.

Our two pedigrees with autosomal dominant inheritance of the TBP repeat expansion are the first familial cases reported thus far. The disease is associated with an elongation of an unstable (CAG)n element (case II and III) and combined with loss of CAA interruptions in Family I within the complex repetitive sequence. However, it is difficult to decide finally whether the expanded alleles are rare polymorphisms or mutations causing a new polyglutamine disease. To avoid problems of predictive testing at this point of uncertainty, unaffected family members were not investigated.

In addition to the partial duplication in the polyglutamine domain of the TBP gene recently described in a girl with gait
Table 1 Frequencies of normal alleles in patients and control individuals. The number of triplets is given.

\begin{tabular}{rrrrrrrr}
\hline Allele & Triplets & Sporadic & $\%$ & Familial & $\%$ & Control & $\%$ \\
\hline 1 & 44 & 1 & 0.10 & & & & \\
2 & 42 & 3 & 0.32 & 1 & 0.37 & & \\
3 & 40 & 14 & 1.50 & 4 & 1.48 & 2 & 0.64 \\
4 & 39 & 86 & 9.17 & 22 & 8.15 & 30 & 9.55 \\
5 & 38 & 382 & 40.72 & 116 & 42.96 & 117 & 37.26 \\
6 & 37 & 205 & 21.85 & 67 & 24.81 & 68 & 21.66 \\
7 & 36 & 161 & 17.16 & 36 & 13.33 & 59 & 18.79 \\
8 & 35 & 57 & 6.07 & 22 & 8.15 & 22 & 7.01 \\
9 & 34 & 4 & 0.43 & & & & \\
10 & 33 & & & & & 1 & 0.32 \\
11 & 32 & 12 & 1.28 & 2 & 0.74 & 6 & 1.91 \\
12 & 31 & 3 & 0.32 & & & 3 & 0.95 \\
13 & 30 & 8 & 0.58 & & & 4 & 1.27 \\
14 & 29 & 1 & 0.10 & & & 2 & 0.64 \\
15 & 28 & & & & & & \\
16 & 27 & 1 & 0.10 & & & & \\
\hline
\end{tabular}

disturbance and intellectual deterioration, ${ }^{8}$ two different genotypes have been now identified that are associated with a neurodegenerative condition. In expanded alleles the CAA interruptions are still present in the $5^{\prime}$ and $3^{\prime}$ regions of the repeat. Such complex structures have not been described for CAG expansions in other genes. Therefore, our results underline the pathological potential of extended polyglutamine stretches independent of the nucleotide sequence and suggest the existence of additional polyglutamine diseases sharing a similar expansion mechanism.

\section{Acknowledgments}

We would like to thank J Atici and U Gehlken for excellent technical help. This work was supported by the Forschungsförderungsprogramm der Medizinischen Universität Lübeck (1799/N03) and the Fritz Thyssen Stiftung, Köln (AZ 1999 2060). We thank all patients for providing blood samples for scientific research and their physicians for collecting them. We thank the German Heredo-Ataxia Society (DHAG), whose cooperation is essential in our work.

\section{References}

1 Cummings CJ, Zoghbi HY: Fourteen and counting: unraveling trinucleotide repeat diseases. Hum Mol Genet 2000; 9: 909-916.

2 Gostout B, Liu Q, Sommer SS: Cryptic repeating triplets of purines and pyrimidines (cRRY(i)) are frequent and polymorphic: analysis of coding cRRY(i) in the proopiomelanocortin (POMC) and TATA-binding protein (TBP) genes. Am J Hum Genet 1993; 52: $1182-1190$.

3 Polymeropoulos MH, Rath DS, Xiao H, Merril CR: Trinucleotide repeat polymorphism at the human transcription factor IID gene. Nucl. Acids Res 1991; 19: 4307.

4 Hoffmann A, Sinn E, Yamamoto T et al: Highly conserved core domain and unique $\mathrm{N}$ terminus with presumptive regulatory motifs in a human TATA factor (TFIID). Nature 1990; 346: 387 390.

5 Kao CC, Lieberman PM, Schmidt MC, Zhou Q, Pei R, Berk AJ: Cloning of a transcriptionally active human TATA binding factor. Science 1990; 248: 1646-1650. 
6 Peterson MG, Tanese N, Pugh BF, Tjian R: Functional domains and upstream activation properties of cloned human TATA binding protein. Science 1990; 248: $1625-1630$.

7 Imbert G, Trottier Y, Beckmann J, Mandel JL: The gene for the TATA binding protein (TBP) that contains a highly polymorphic protein coding CAG repeat maps to 6q27. Genomics 1994; 21 : $667-668$

8 Koide R, Kobayashi S, Shimohata T et al: A neurological disease caused by an expanded CAG trinucleotide repeat in the TATAbinding protein gene: a new polyglutamine disease? Hum Mol Genet 1999; 8: 2047-2053.
9 Schöls L, Gispert S, Vorgerd M et al: Spinocerebellar ataxia type 2. Arch Neurol 1997; 54: 1073-1080.

10 Lerer I, Merims D, Abeliovich D, Zlotogora J, Gadoth N: Machado-Joseph Disease: Correlation between clinical features, the CAG repeat length and homozygosity of the mutation. Eur $J$ Hum Genet 1996; 4: 3-7.

11 Warner TT, Williams LD, Walker RWH et al. A clinical and molecular genetic study of DRPLA in four European families. Ann Neurol 1995; 37: 452-459. 